\title{
Stored energy of arbitrary metamaterial inclusions
}

\author{
Ozuem Chukwuka, a) Divitha Seetharamdoo, b) and M. Hassanein Rabah \\ Univ. Lille Nord de France, IFSTTAR, COSYS, LEOST F-59650 Villeneuve d'Ascq, France.
}

(Dated: 24 July 2020)

\section{INTRODUCTION}

Electromagnetic systems such as antennas are char- ${ }_{55}$ acterised by the way in which they dissipate, store and radiate electromagnetic energy. These quantities are useful for understanding the physics, design application and evaluation of the radiation performances such as the radiation efficiency and performance bounds ${ }^{1-4}$. It is gen- 60 erally suggested that electromagnetic artificial materials such as metamaterial can improve radiating performances in several configurations ${ }^{5}$. These metamaterials (MTMs) are defined in terms of their effective material parameters i.e. permeability $\mu$ and permittivity $\varepsilon$ func- ${ }_{65}$ tions and always need to be extracted ${ }^{6}$ from their farfield quantity. Importantly, an interesting phenomena for electromagnetic systems application happen at the region of resonance ${ }^{7,8}$ where effective parameters are not always valid. Hence, with the growing near-field applications of ${ }_{70}$ MTM to electromagnetic systems like antenna ${ }^{9,10}$, it is o interesting to analyze MTM around its region of resonance using similar quantities of most electromagnetic systems. Unlike effective parameters which are valid in far-field, stored energy quantities define near-field inter- ${ }_{75}$ action of electromagnetic systems ${ }^{11}$. Although, there are existing methods for evaluating electromagnetic energy such as the scattering approach given in terms of the conduction or polarization current ${ }^{12}$, a more robust and intuitive approach is the method of moments ${ }^{13}$.

The method of moments which is applied in the theory of characteristic modes (TCM) brings physical insight through intuitive knowledge into the electromagnetic energy of radiating structures using a modal approach ${ }^{14}$.
It expands current as a number of basis function and green function and by solving the derived impedance matrix as a generalized eigenvalue equation, electromagnetic energy can be calculated for any external exciting field. In other words, the application of theory of characteristic modes is independent of excitation, applicable to arbitrary-shaped structures, takes into account the nature of the material and requires only few modes to describe the global behaviour of electrically small structures ${ }^{14}$. Thus, TCM is popular for the design of many electromagnetic systems especially antenna design ${ }^{15-18}$ for various applications. In previous work, the theory of characteristic modes was extended to design MTM inclusion ${ }^{19}$. It was shown that one of the challenges with these inclusions especially magnetic inclusion is that its magnetic properties are exhibited when excitation is placed in a specific polarization. Thus, it is important to consider excitation and polarization effect in describing the modal stored energy of MTM inclusion.

The modal stored energy approach for metamaterial inclusion is useful for selecting an inclusion to associate with an electromagnetic system like antenna. Already, many electromagnetic designs make use of modal approach such as evaluation of antenna Q-factor ${ }^{20-22}$, selection of antenna excitation source ${ }^{23}$, design of metamaterial inclusion for antenna application ${ }^{24-27}$ and the study of electromagnetic structures ${ }^{28-30}$. This is due to the numerous advantages which include its independence of excitation source, applicability to arbitrary-shaped structures and the physical insight it brings into the behaviour of radiating structures ${ }^{14,15}$. The design of metamaterial, antenna and electromagnetic devices can therefore be an- 
yzed using similar modal quantities while leveraging on the physical insight and other advantages of modal analysis approach.

This paper shows a method to quantitatively evaluate the modal stored energy of MTM inclusions using TCM. count the properties and structure of the material. The methodology is applied to MTM inclusions with known effective parameter quantity. In order to provide a qualitative appreciation by comparison to common effective energy approach to account for the polarization and excitation dependence of MTM inclusions. Two elementary structures, broadside-coupled split-ring resonator (BC$\mathrm{SRR}$ ) and S-shaped unit cell are considered to demoncour approach. The commercial method of moment (MoM) based characteristic modes analysis tool $(\mathrm{FEKO})^{31}$ is used to evaluate the eigenvalue, modal surface current distribution and modal weighting coefficient. The impedance matrix is extracted and used to evaluate the stored energy.

\section{FORMULATION FOR MODAL STORED ENERGY EVALUATION BASED ON THEORY OF CHARACTERISTIC MODES}

The modal stored energy formulation is based on the Theory of Characteristic Modes (TCM) which representıз a structure in terms of its surface impedance $\mathrm{Z}^{14}$. The surface impedance $\mathrm{Z}$ is given as:

$$
\mathrm{Z}=\mathrm{R}+\mathrm{jX}
$$

where $\mathrm{R}$ is the real part and $\mathrm{X}$ is the imaginary part of the impedance. Using the derived impedance Z, a solution is found for the generalized eigenvalue equation given in equation (2) as:

$$
[\mathrm{X}][\mathrm{I}]=\lambda_{\mathrm{n}}[\mathrm{R}][\mathrm{I}] \text {, }
$$

where $\lambda_{\mathrm{n}}$ is the eigenvalue of each $\mathrm{n}$ mode and $\mathrm{I}$ is the eigen-current.

Although this paper uses TCM based method for the presented formulation of the modal stored energy, TCM is not explicitly dealt here and can be found in the literature $^{14,15,32,33}$. The value of $\lambda_{\mathrm{n}}$ is directly related to the nature of the modal stored energy by the imaginary part of the impedance matrix $\mathrm{X}$ with $\lambda_{\mathrm{n}}$ greater than zero indicating a stored magnetic energy, $\lambda_{\mathrm{n}}$ less than zero indicating a stored electric energy and $\lambda_{\mathrm{n}}$ equal to zero indicating that both the magnetic energy and electric energy are of equal magnitude which results in total radiation with no stored energy ${ }^{2,15,34}$. This modal stored energy deductions are however only qualitative ${ }^{14}$. On the other hand, modal quantitative stored energy value of MTM inclusion provides a new way to reliably analyze artificial materials. This would aid their choice for specific applications especially in cases where the near-field behaviour needs to be predicted such as its association to antennas ${ }^{10}$ and sensors.
Using the surface impedance $\mathrm{Z}$ derived from TCM, the modal stored energy operator $\left(\mathrm{W}_{\text {sto }}\right)$ derived in ${ }^{35,36}$ and as applied to antenna design ${ }^{2}$ is:

$$
\mathrm{W}_{\text {sto }}=\mathrm{W}_{\mathrm{m}}+\mathrm{W}_{\mathrm{e}}=\frac{1}{4 \omega} \mathrm{I}^{\mathrm{H}} \mathrm{X}^{\prime} \mathrm{I},
$$

where $\omega$ is the angular frequency, I is the eigen-current from the generalized eigenvalue equation, $\mathrm{I}^{\mathrm{H}}$ is the Hermitian transpose of the eigen-current and $\mathrm{X}^{\prime}$ is given $\mathrm{in}^{2}$ as equation (4):

$$
\mathrm{X}^{\prime}=\omega \frac{\partial \mathrm{X}}{\partial \omega} .
$$

Separating $\mathrm{W}_{\text {sto }}$ into electric energy $\left(\mathrm{W}_{\mathrm{e}}\right)$ and magnetic energy $\left(\mathrm{W}_{\mathrm{m}}\right)$ component $^{2}$, we have equation (5) and (6):

$$
\mathrm{W}_{\mathrm{m}}=\frac{1}{8 \omega} \mathrm{I}^{\mathrm{H}} \mathrm{X}_{\mathrm{m}} \mathrm{I},
$$

and

$$
\mathrm{W}_{\mathrm{e}}=\frac{1}{8 \omega} \mathrm{I}^{\mathrm{H}} \mathrm{X}_{\mathrm{e}} \mathrm{I}
$$

where,

$$
\mathrm{X}_{\mathrm{m}}=\mathrm{X}^{\prime}+\mathrm{X}
$$

and

$$
\mathrm{X}_{\mathrm{e}}=\mathrm{X}^{\prime}-\mathrm{X}
$$

$\mathrm{X}$ being a 3D matrix complicates the implementation of $\mathrm{X}^{\prime}$, thus further simplification is carried out by splitting the impedance $\mathrm{Z}$ into its inductive and capacitive component as:

- For X as an inductive impedance:

$$
\mathrm{X}=\omega L
$$

differentiating both sides with respect to $\omega$,

$$
\frac{\partial \mathrm{X}}{\partial \omega}=L
$$

But from equation (4),

$$
\mathrm{X}^{\prime}=\omega \frac{\partial \mathrm{X}}{\partial \omega}
$$

substituting equation (10) into equation (4),

$$
\mathrm{X}^{\prime}=\omega \frac{\partial \mathrm{X}}{\partial \omega}=\omega L
$$

therefore substituting equation (9) into equation (11),

$$
\mathrm{X}^{\prime}=\mathrm{X}
$$

- For $\mathrm{X}$ as a capacitive impedance:

$$
\mathrm{X}=\frac{-1}{\omega C},
$$

differentiating both sides with respect to $\omega$,

$$
\frac{\partial \mathrm{X}}{\partial \omega}=\frac{1}{C \omega^{2}} \text {. }
$$


But from equation (4),

$$
\mathrm{X}^{\prime}=\omega \frac{\partial \mathrm{X}}{\partial \omega}
$$

substituting equation (14) into equation (4),

$$
\mathrm{X}^{\prime}=\omega \frac{\partial \mathrm{X}}{\partial \omega}=\omega \frac{1}{C \omega^{2}}=\frac{1}{C \omega},
$$

therefore substituting equation (13) into equation (15),

$$
\mathrm{X}^{\prime}=-\mathrm{X}
$$

The conclusion therefore is that $\mathrm{X}^{\prime}$ equals $|\mathrm{X}|$ and the two possible analytical solution for $\mathrm{X}^{\prime}$ depends on the nature of $\mathrm{W}_{\text {sto }}$ as either electric or magnetic. This conclusion has been qualitatively concluded using the first derivative of $\lambda_{\mathrm{n}}$ with respect to the frequency ${ }^{21}$.

To ensure uniformity of the modal stored energy value, just as it is done for $W_{\text {sto }}$ in antenna applications for evaluation of $\mathrm{Q}$-factor, $\mathrm{W}_{\mathrm{m}}$ and $\mathrm{W}_{\mathrm{e}}$ are normalized with respect to the radiated power $\left(\mathrm{P}_{\mathrm{r}}\right)^{2,20,37}$ in equation (17):

$$
\mathrm{P}_{\mathrm{r}}=\mathrm{I}^{\mathrm{H}} \mathrm{RI}
$$

\section{EVALUATION AND ANALYSIS OF MODAL STORED ENERGY OF MTM INCLUSION}

In this section, the $\mathrm{W}_{\text {sto }}$ formulation is applied to MTM inclusions to determine their modal magnetic and electric energies. The difference between the magnetic and electric energy $\left(\mathrm{W}_{\mathrm{m}}-\mathrm{W}_{\mathrm{e}}\right)$ is used to determine ishing energy (resonance) ${ }^{34}$. Two elementary inclusions with known effective parameters are considered; the Broadside-Coupled Split-Ring Resonator (BC-SRR) and the S-shaped inclusion. While the BC-SRR is a well the S-shaped inclusion exhibit both the electric and magnetic behaviour at different resonant frequencies. The analysis for the structures are done in the frequency range of $1 \mathrm{GHz}$ to $4 \mathrm{GHz}$ to cover the resonance of both inclusions.

\section{III.A. Broadside-Coupled Split-Ring Resonator (BCSRR)}

The BC-SRR which is known to exhibit artificial magnetism is made up of two circular symmetric loops of PEC material and placed on the two opposite sides of a teflon substrate of effective permittivity, $\epsilon_{r} 2.2$. It resonate at $2.35 \mathrm{GHz}$ and its dimension is given in figure 1 .

The artificial magnetic behavior of this BC-SRR was shown by Rabah et al. ${ }^{19}$ using characteristic modes and static polarizability. The TCM commercial software was used for the modal analysis of the structure to solve the generalized eigenvalue equation and the obtained result of the TCM analysis for the structure is represented byiz5

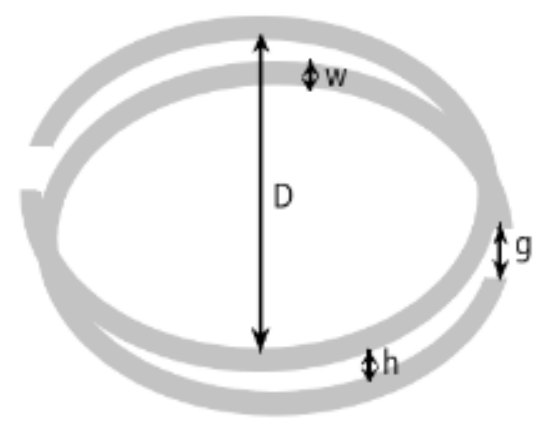

FIG. 1: BC-SRR with dimensions: $\mathrm{D}=11.25 \mathrm{~mm}, \mathrm{~W}=$ $1 \mathrm{~mm}, \mathrm{~g}=1.95 \mathrm{~mm}, \mathrm{~h}=$ substrate height $=1.08 \mathrm{~mm}$.

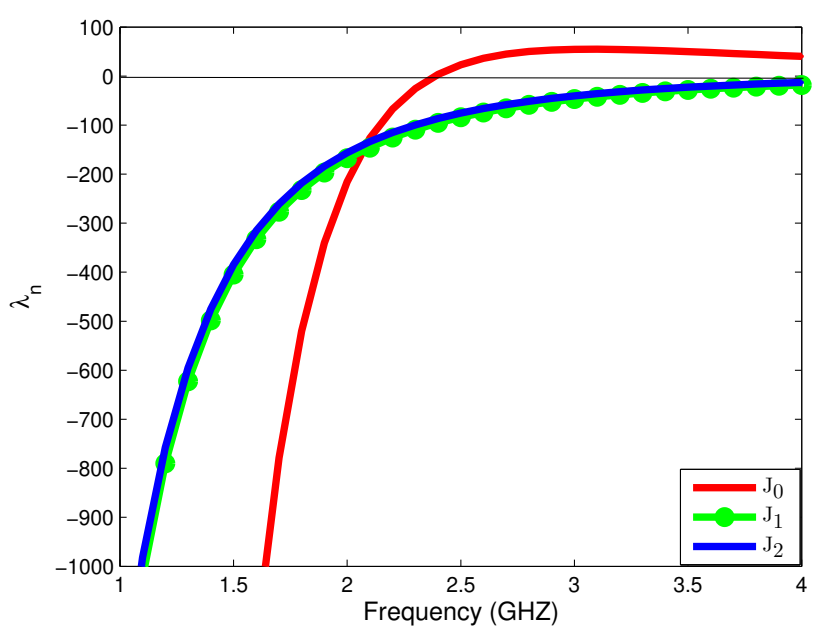

(a)

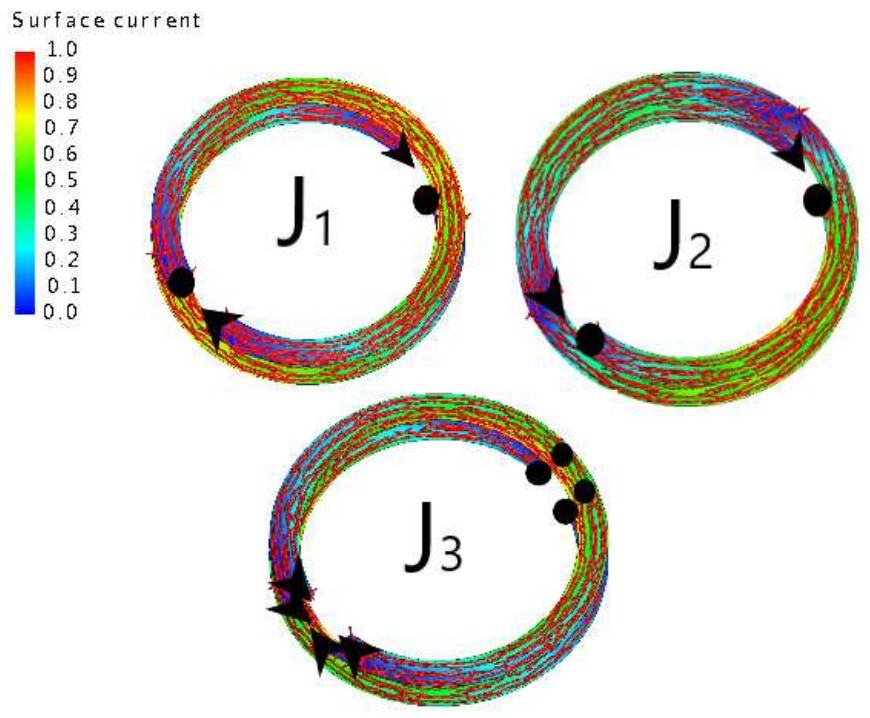

(b)

FIG. 2: BC-SRR inclusion: (a)eigenvalue $\left(\lambda_{\mathrm{n}}\right)$ with respect to frequency (b)surface current distribution

the plot of $\lambda_{\mathrm{n}}$ with respect to frequency in figure $2 \mathrm{a}$ and the surface current distribution in figure $2 \mathrm{~b}$. 
Only the fundamental mode $J_{0}$ in red cross the zeron10 mark at $2.35 \mathrm{GHz}$ as shown in figure 2a hence, only one resonance occur. The $\mathrm{W}_{\mathrm{e}}$ and $\mathrm{W}_{\mathrm{m}}$ are of equal magnitude and no energy is stored at this frequency of 2.35 $\mathrm{GHz}$. Also, this resonance frequency of $2.35 \mathrm{GHz}$ is close to that of the BC-SRR analyzed by Rabah et al. ${ }^{19}$ which was referred to as the fundamental DC mode of a circular loop. The other modes $J_{1}$ and $J_{2}$ in green and blue have negative values of $\lambda_{\mathrm{n}}$ through out the considered frequency band and indicates that they store more $\mathrm{W}_{\mathrm{e}}$. From the current profile shown in figure $2 b$, the black arrow head show the direction of the current flow, the black dot show the starting point of the current flow. The behaviour of the modes can be easily described as magnetic ectric. $J_{0}$ show a magnetic current distribution with both loops having similar direction of current flow while $J_{1}$ and $J_{2}$ show a current distribution pattern similar to that of an electric dipole with the current flowing in the opposite direction on both loops.

To quantify the energies with respect to frequency for each mode, the impedance matrix is extracted from the commercial software and the $\mathrm{W}_{\text {sto }}$ formulation of section II is applied to determine the modal magnetic and electric energies. The result of the dominant energy is represented by the normalized $\left(\mathrm{W}_{\mathrm{m}}-\mathrm{W}_{\mathrm{e}}\right)$ with respect to frequency in figure 3.

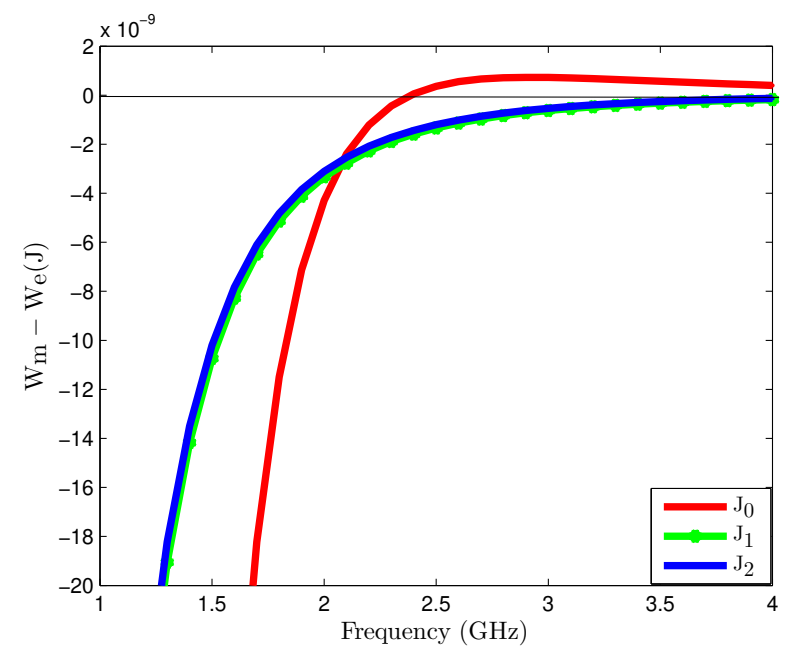

FIG. 3: Normalized $\left(\mathrm{W}_{\mathrm{m}}-\mathrm{W}_{\mathrm{e}}\right)$ with respect to frequency of BC-SRR.

The value of $\mathrm{W}_{\mathrm{e}}$ and $\mathrm{W}_{\mathrm{m}}$ are equal at $2.35 \mathrm{GHz}$ for mode $J_{0}$ in red thus, the value of normalized $\left(\mathrm{W}_{\mathrm{m}}-\mathrm{W}_{\mathrm{e}}\right)$ is $0 \mathrm{~J}$ at this frequency. $J_{1}$ and $J_{2}$ in green and blues45 both have negative values of $\left(\mathrm{W}_{\mathrm{m}}-\mathrm{W}_{\mathrm{e}}\right)$ within the considered frequency band and implies that $\mathrm{W}_{\mathrm{e}}$ remains greater than $\mathrm{W}_{\mathrm{m}}$. The $\left(\mathrm{W}_{\mathrm{m}}-\mathrm{W}_{\mathrm{e}}\right)$ quantity agrees with the qualitative deductions of the eigenvalue $\left(\lambda_{\mathrm{n}}\right)$ curve.

\section{III.B. S-shaped MTM inclusion}

The S-shaped inclusion exhibit both the electric and magnetic behaviour due to its bianisotropic character ${ }^{38}$. It is made of a PEC sheet forming an S-shape and its dimensions are shown in figure 4.

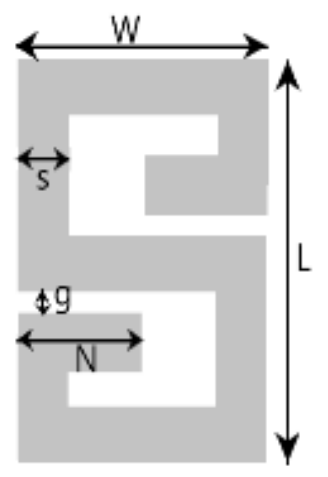

FIG. 4: S-shaped inclusion with dimensions: $\mathrm{L}=27$ $\mathrm{mm}, \mathrm{W}=13 \mathrm{~mm}, \mathrm{~g}=1.1 \mathrm{~mm}, \mathrm{~N}=4 \mathrm{~mm}, \mathrm{~s}=1.5 \mathrm{~mm}$.

The S-shaped structure has its fundamental resonance at $1.92 \mathrm{GHz}$. The same analysis followed for the BC-SRR inclusion was carried out for the S-shaped inclusion. The TCM commercial software was used for the modal analysis of the structure and the obtained result of the TCM 220 frequency in figure $5 \mathrm{a}$ and the surface current distribution in figure $5 \mathrm{~b}$.

$J_{0}$ and $J_{2}$ of figure $5 \mathrm{a}$ in red and blue cross the zero mark at $1.92 \mathrm{GHz}$ and $3.1 \mathrm{GHz}$ respectively. This corresponds to the resonance frequencies and indicates that $\mathrm{W}_{\mathrm{e}}$ and $\mathrm{W}_{\mathrm{m}}$ are of equal magnitude at these frequencies and no modal stored energy. $J_{1}$ on the other hand remains with negative values of $\lambda_{\mathrm{n}}$ with respect to the considered frequency band implying that $\mathrm{W}_{\mathrm{e}}$ is dominant. ehaviour of the modes as electric or magnetic can also be analyzed using the surface current distribution in figure 5b. The black arrow head show the direction of the current flow and the black dot shows its starting point. The current distribution of $J_{0}$ is similar to that of an electric dipole with the current flowing in one direction. $J_{1}$ also show an electric dipole current distribution splitted into two halves with the current flowing in opposite directions on the two halves. $J_{2}$ show a magnetic current distribution with the current flowing in the same direction when the S-shape is split into two equal halves.

To quantify the modal energies with respect to frequency for each mode, the impedance matrix is extracted from the commercial software and the $\mathrm{W}_{\text {sto }}$ formulation of section II is applied to determine the modal magnetic and electric energies. The result of the dominant energy is represented by the normalized $\left(\mathrm{W}_{\mathrm{m}}-\mathrm{W}_{\mathrm{e}}\right)$ with respect to frequency in figure 6 .

$J_{0}$ and $J_{2}$ of figure 6 in red and blue show that at the resonance frequency of $1.92 \mathrm{GHz}$ and $3.1 \mathrm{GHz}$ respectively, $\left(\mathrm{W}_{\mathrm{e}}-\mathrm{W}_{\mathrm{m}}\right)$ is $0 \mathrm{~J}$ and the magnitude of $\mathrm{W}_{\mathrm{m}}$ and 


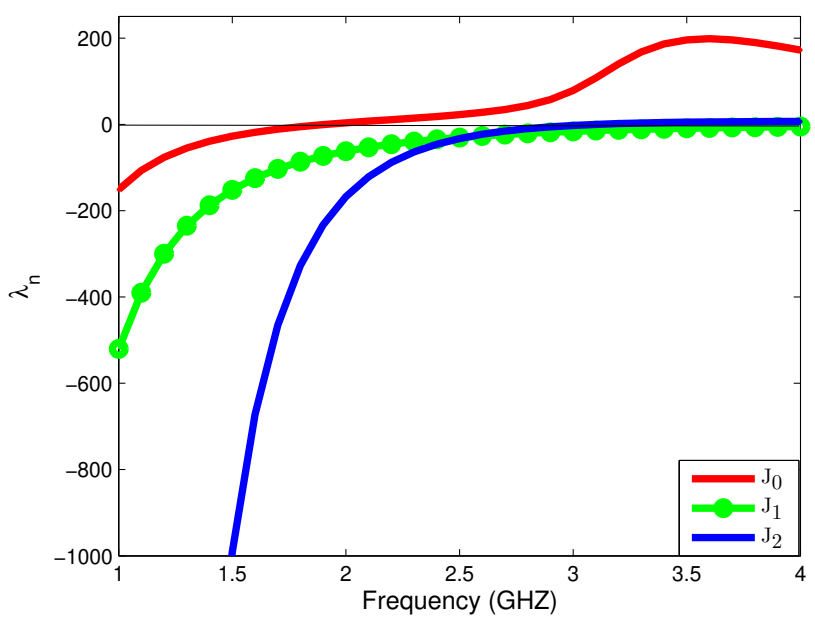

(a)

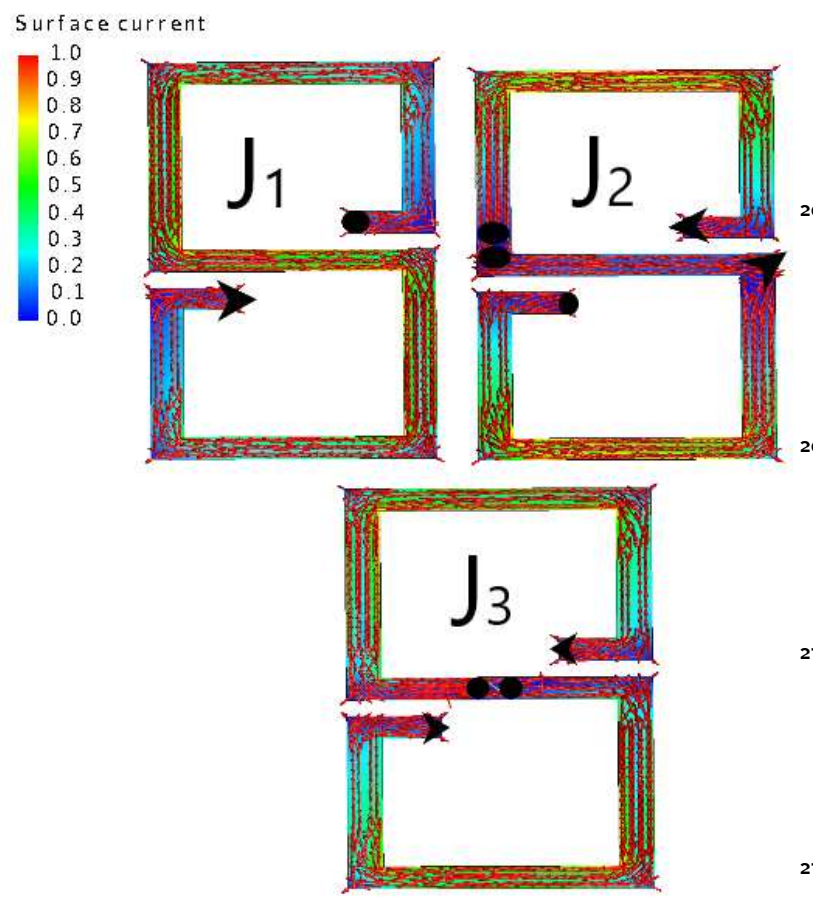

(b)

FIG. 5: S-shaped inclusion: (a)eigenvalue $\lambda_{\mathrm{n}}$ with respect to frequency (b)surface current distribution

$\mathrm{W}_{\mathrm{e}}$ are of the same value. The region of the curve that lies in the negative part indicates that $\mathrm{W}_{\mathrm{e}}$ is dominant while the region in the positive part indicates that $\mathrm{W}_{\mathrm{m}}$ is dominant. $J_{1}$ in green has negative values across thezoo considered frequency band hence, $\mathrm{W}_{\mathrm{e}}$ is dominant across the considered frequency band. The $\left(\mathrm{W}_{\mathrm{m}}-\mathrm{W}_{\mathrm{e}}\right)$ quantity agrees with the qualitative deductions of the eigenvalue $\left(\lambda_{\mathrm{n}}\right)$ curve.

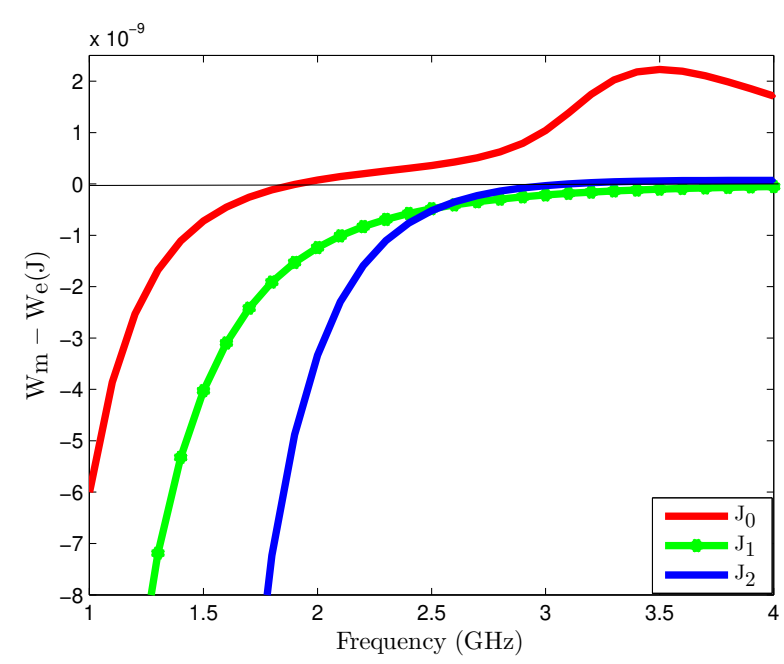

FIG. 6: Normalized $\left(\mathrm{W}_{\mathrm{m}}-\mathrm{W}_{\mathrm{e}}\right)$ with respect to frequency of S-shaped inclusion.

\section{COMPARISON BETWEEN STORED ENERGY AND EFFECTIVE PARAMETERS OF MTM INCLUSION}

Generally, the physical behaviour of MTM are described in terms of effective parameters ${ }^{39-42}$. The later are calculated from far-field complex reflection and transmission coefficient ${ }^{41,42}$ using classical approach of extraction techniques ${ }^{40,43,44}$. The effective parameters of MTM structures are represented as the permittivity $\varepsilon$ and permeability $\mu$ values which describes them as electric or magnetic $\mathrm{MTM}^{45}$. If this provides physical insight, the 270 association of MTM to devices at microwave frequencies in the near-field region such as for antenna and sensor designs $^{46-48}$ can not be studied in a reliable manner based on effective parameters.

Modal stored energy on the other hand is a quantity 275 derived from modal current and account for near-field effects $^{1}$. It permits studying the behaviour of shapes separately from its feeding structure. This means that the modal quantities of the structure is calculated once while the effect of the feeding can be superimposed ${ }^{20,49}$. The 280 modal stored energy being quantitative and independent of excitation is believed to be a more reliable analysis of MTM since the properties of MTM are based on their shapes $^{50}$.

In this section, we present a comparison between the effective parameter and the dominant stored energy such that within a given frequency band, one can appreciate the convergence in terms of physical and qualitative analysis. Most inclusions especially magnetic inclusion have their magnetic properties exhibited when excitation is placed in a specific polarization ${ }^{19}$. The TCM based energies $W_{m}$ and $W_{e}$ are excitation and polarization independent whereas, the effective parameters are excitation and polarization dependent. To enable a comparison between the two approach, new steps are introduced to the 

- W ) to account for the polarizability of the inclusions. Additional steps are introduced to the calculation of $\left(\mathrm{W}_{\mathrm{m}}\right.$ $-\mathrm{W}_{\mathrm{e}}$ ) in figure 7 .

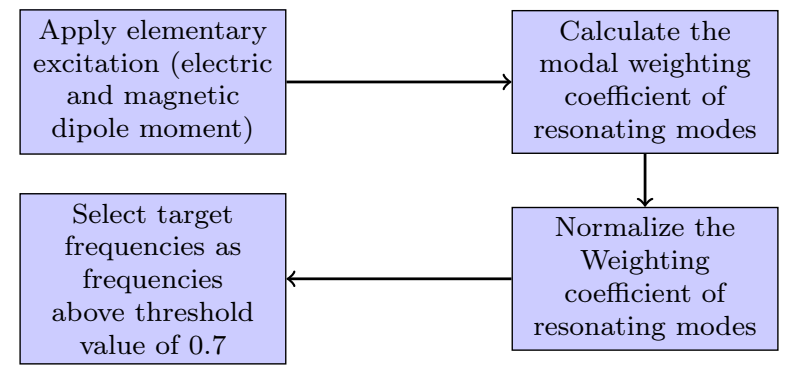

Fig. 7: Additional steps for calculating $\left(\mathrm{W}_{\mathrm{m}}-\mathrm{W}_{\mathrm{e}}\right)$.

The block diagram in figure 7 describes the procedure to take into account the effect of excitation and polarization in the modal stored energy analysis of MTM inclusion. First, an elementary excitation (electric and magnetic dipole moment) is applied to the structure and the modal weighting coefficient $\mathrm{MWC}_{\mathrm{n}}{ }^{14}$ is derived from the as equation (18):

$$
\mathrm{MWC}_{\mathrm{n}}=\frac{\mathrm{V}^{\mathrm{i}} \mathrm{J}_{\mathrm{n}}}{1+j \lambda_{\mathrm{n}}}
$$

where $\mathrm{V}^{\mathrm{i}}$ is the applied excitation, $\lambda_{\mathrm{n}}$ is the eigenvalue and $\mathrm{J}_{\mathrm{n}}$ is the surface current of the $\mathrm{n}^{\text {th }}$ mode.

The $\mathrm{MWC}_{\mathrm{n}}$ give information of the modal response $3_{45}$ to the applied excitation. The $\mathrm{MWC}_{\mathrm{n}}$ of the resonant modes are normalized with their maximum value. Stored energy is related to bandwidth by Q-factor definition ${ }^{51,52}$ hence, we evaluate the modal half power bandwidth using the TCM bandwidth definition. This represents thesso is given as frequencies with a modal sionificance above $0.7^{15}$. The dominant energy within the modal bandwidth (i.e. having a value above 0.7 of the normalized $\mathrm{MWC}_{\mathrm{n}}$ ) are considered and referred to as target frequencies. They $y_{355}$ frequencies which are important for electromagnetic applications. To quantitatively describe an inclusion, the dominant energy $\left(\mathrm{W}_{\mathrm{m}}-\mathrm{W}_{\mathrm{e}}\right)$ within the target frequencies are summed up and the sign of the summation indicates the dominant energy within the modal bandwidth of the structure. It tells if the inclusion is electric or mag.360 netic. A positive value, indicates dominant $\mathrm{W}_{\mathrm{m}}$ and the inclusion is magnetic while a negative value, implies that $\mathrm{W}_{\mathrm{e}}$ is dominant and the inclusion is electric.

This procedure is applied to the inclusions whose dominant modal energy values $\left(\mathrm{W}_{\mathrm{m}}-\mathrm{W}_{\mathrm{e}}\right)$ were evaluated $\mathrm{in}_{\mathbf{6} 65}$ the previous section.

\section{IV.A. Broadside Coupled Split Ring Resonator (BCSRR)}

The BC-SRR is excited with a magnetic dipole moment and only the resonant mode $J_{0}$ is considered. The normalized $\mathrm{MWC}_{\mathrm{n}}$ with respect to frequency extracted from the TCM based simulation software is shown in fig. 8.

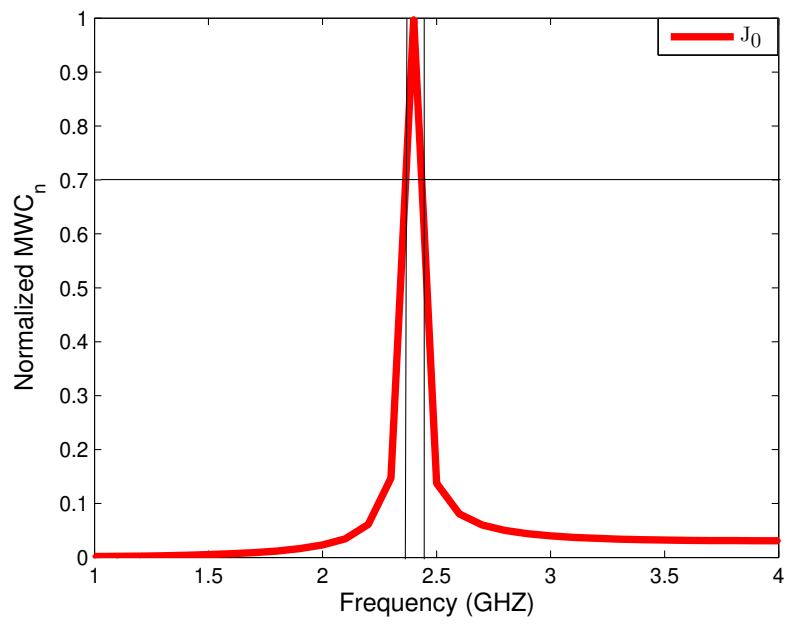

FIG. 8: Normalized $\mathrm{MWC}_{\mathrm{n}}$ with respect to frequency of BC-SRR.

The horizontal thin black line in figure 8 show the threshold value of 0.7 which indicates the region of the target frequencies between $2.35 \mathrm{GHz}$ and $2.43 \mathrm{GHz}$. The shift of the centre frequency from the resonance is due to the coupling of the excitation with the structure. The summation of the normalized $\left(\mathrm{W}_{\mathrm{m}}-\mathrm{W}_{\mathrm{e}}\right)$ at the target frequencies from the previous section for the BC-SRR is $4.1969 \times 10^{-10} \mathrm{~J}$. The value is positive hence, one can conclude that $J_{0}$ of BC-SRR exhibits a magnetic behaviour and therefore it is a magnetic inclusion. This analysis agree with the current profile of BC-SRR. In comparison to the classical approach of effective parameters of the BC-SRR, the plot of effective parameters $(\varepsilon$ and $\mu)$ with respect to frequency is shown in figure 9 .

$\varepsilon$ and $\mu$ in green and red respectively give information of the dominant property of the BC-SRR. Around the resonance frequency of $2.35 \mathrm{GHz}$, the values of $\mu$ are higher than that of $\varepsilon$ and the magnetic behaviour of the structure dominates thus, the inclusion is magnetic. This qualitative description is in agreement with that of the dominant stored energy analysis.

\section{IV.B. S-shaped MTM inclusion}

The S-shaped inclusion has two resonant modes $J_{0}$ and $J_{2}$ with different behavior. The S-shaped inclusion is excited with an electric dipole moment and the normalized $\mathrm{MWC}_{\mathrm{n}}$ with respect to frequency which has been extracted from the TCM based simulation software is shown in figure 10. 


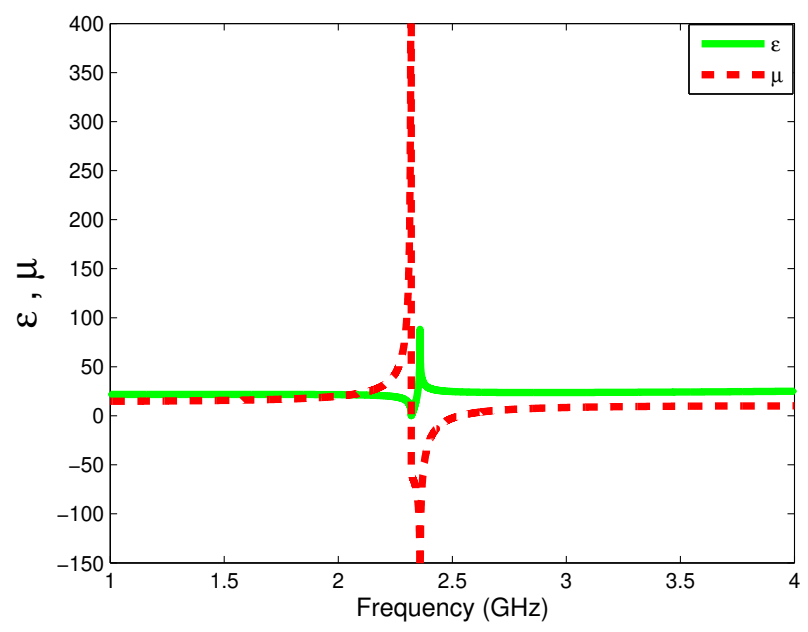

FIG. 9: $\varepsilon$ and $\mu$ with respect to the frequency of BC-SRR.

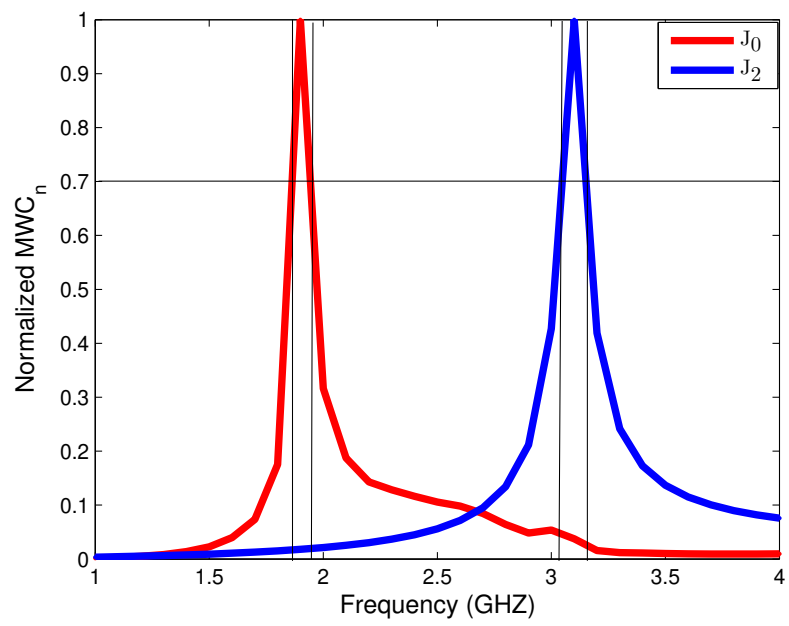

FIG. 10: Normalized $\mathrm{MWC}_{\mathrm{n}}$ with respect to frequency of S-shaped inclusion.

The horizontal thin black line in figure 10 show the threshold value of 0.7 which indicates the region of the target frequencies between $1.86 \mathrm{GHz}$ and $1.94 \mathrm{GHz}$ for mode $J_{0}$ in red and between $3.05 \mathrm{GHz}$ and $3.2 \mathrm{GHz}$ for 405 mode $J_{2}$ in blue. The shift of the centre frequency from the resonance frequency is due to the coupling of the excitation with the structure. The summation of the $\left(\mathrm{W}_{\mathrm{m}}-\mathrm{W}_{\mathrm{e}}\right)$ values at the target frequencies from the previous section for the S-shaped inclusion is $-1.2 \times 10^{-10} \mathrm{~J}_{410}$ and $2.5545 \times 10^{-11} \mathrm{~J}$ for $J_{0}$ and $J_{2}$ respectively. The value for $J_{0}$ is negative indicating that the inclusion behave as an electric structure around the first resonance. $J_{2}$ has a positive value which implies that the inclusion behave

as a magnetic structure at its second resonance. Thisa15 analysis agree with the current profile of the S-shaped inclusion. In comparison to the classical approach of effective parameters of the S-shaped inclusion, the plot of effective parameters $(\varepsilon$ and $\mu$ ) with respect to frequency is shown in figure 11 .

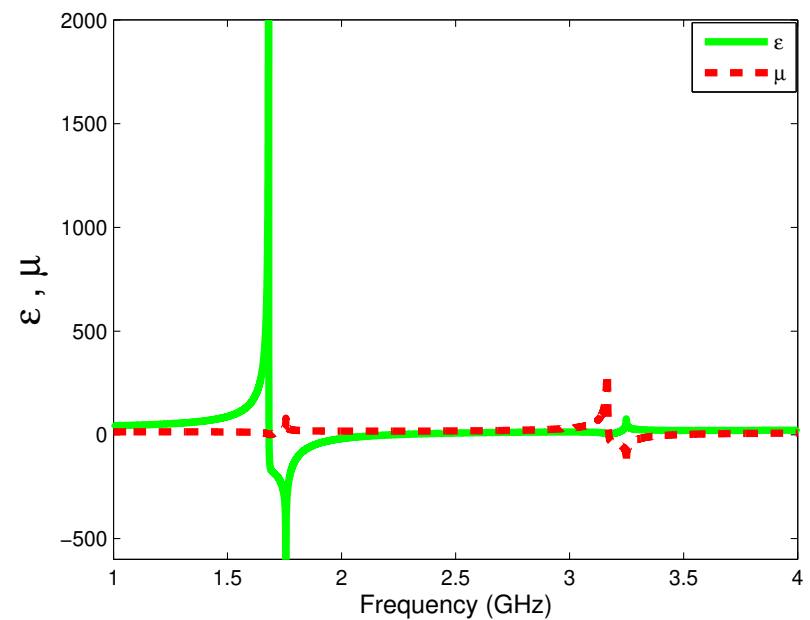

FIG. 11: $\varepsilon$ and $\mu$ with respect to the frequency of S-shaped inclusion.

$\varepsilon$ and $\mu$ in green and red respectively give information of the dominant property of the S-shaped inclusion. Around the fundamental resonance frequency of $1.92 \mathrm{GHz}$, the values of $\varepsilon$ are higher than that of $\mu$ and 390 the electric behaviour dominates the magnetic behaviour hence, the structure behave as an electric inclusion. On the other hand, the values of $\mu$ are higher than that of $\varepsilon$ at the second resonance of $3.01 \mathrm{GHz}$ and the structure behave as a magnetic inclusion at this frequency. This qualitative description is in agreement with that of the dominant stored energy analysis for the S-shaped inclusion.

\section{CONCLUSION}

In comparison to the effective parameters approach of defining metamaterial inclusions which are valid in the far-field region and may not be reliable for nearfield analysis and modal design of electromagnetic devices, this paper presents a modal stored energy approach which is based on modal current and account for nearfield effects. This method can be applied to any resonant metamaterial structure and it is based on the theory of characteristics modes which is independent of excitation and brings physical insight into the resonant property of a structure. Two elementary inclusions, BC-SRR and S-shaped inclusions were considered for demonstration. Also, a comparison between the effective parameters and the modal stored energy approach is done to show the convergence in terms of physical and qualitative analysis. This comparison is achieved by introducing additional steps into the calculation of modal stored energy to take into account the polarization and excitation dependence of inclusions. Both methods show good agreement in their physical and qualitative analysis. This approach 
is useful in the application of modal analysis for design-485 ing metamaterial-inspired structures including antenna, sensor and cloaks.

\section{ACKNOWLEDGMENTS}

The authors acknowledge partial funding of this research work by the regional project SMARTIES in the framework of the ELSAT 2020 program co-financed by the European Union with the European Regional development fund, the French state and Hauts de France Regional council. One of the author acknowledge funding of IFSTTAR for a PhD scholarship.

a) Electronic mail: ozuem.chukwuka@ifsttar.fr

b) Electronic mail: divitha.seetharamdoo@ifsttar.fr

${ }^{1}$ M. Capek, L. Jelinek, P. Hazdra, and J. Eichler, "The measurablesos q factor and observable energies of radiating structures," IEEE

Transactions on Antennas and Propagation 62, 311-318 (2014).

M. Capekand L. Jelinek, "Optimal composition of modal currents for minimal quality factor $q$," IEEE Transactions on Antennas and Propagation 64, 5230-5242 (2016).

${ }^{3}$ M. Gustafssonand B. Jonsson, "Stored electromagnetic energy and antenna q. eprint," arXiv preprint arXiv:1211.5521 (2013). ${ }^{4}$ M. Gustafsson, "State-space models for stored energy and qfactors," in Electromagnetic Theory (EMTS), 2016 URSI International Symposium on (IEEE, 2016) pp. 226-228.

${ }^{5} \mathrm{D}$. Seetharamdoo, Étude des métamatériaux à indice de réfraction négatif: paramètres effectifs et applications antennaires potentielles, Ph.D. thesis, Rennes 1 (2006).

${ }^{6}$ L. D. Landau, J. Bell, M. Kearsley, L. Pitaevskii, E. Lifshitz, and J. Sykes, Electrodynamics of continuous media, Vol. 8 (elsevier 520 2013).

${ }^{7}$ I. Semchenko, A. Balmakou, S. Khakhomov, and S. Tretyakov, "Stored and absorbed energy of fields in lossy chiral singlecomponent metamaterials," Physical Review B 97, 014432 (2018)

${ }^{8}$ S. Tretyakov, "Electromagnetic field energy density in artificial microwave materials with strong dispersion and loss," physics Letters A 343, 231-237 (2005).

${ }^{9}$ M. H. Rabahand D. Seetharamdoo, "Calculation of the total qfactor for electrically small antennas with metamaterials usings 30 characteristic modes," in 2016 10th European Conference on Antennas and Propagation (EuCAP) (IEEE, 2016) pp. 1-5.

R. W. Ziolkowski, "Applications of metamaterials to realize efficient electrically small antennas," in Antenna Technology: Small Antennas and Novel Metamaterials, 2005. IWAT 2005. IEEF53 International Workshop on (IEEE, 2005) pp. 7-10.

${ }^{11}$ D. Sarkar, S. M. Mikki, A. M. Alzahed, K. V. Srivastava, and Y. M. Antar, "New considerations on electromagnetic energy in antenna near-field by time-domain approach," in 2017 IEEE Applied Electromagnetics Conference (AEMC) (IEEE, 2017) pp540 $1-2$

${ }^{12}$ G. W. Hansonand A. B. Yakovlev, Operator theory for electromagnetics: an introduction (Springer Science \& Business Media, 2013).

${ }^{13} \mathrm{~W}$. C. Gibson, The method of moments in electromagnetic 545 (Chapman and Hall/CRC, 2007).

${ }^{14} \mathrm{M}$. Cabedo-Fabres, E. Antonino-Daviu, A. Valero-Nogueira, and M. F. Bataller, "The theory of characteristic modes revisited: A contribution to the design of antennas for modern applications," IEEE Antennas and Propagation Magazine 49, 52-68 (2007). $\quad 550$

${ }^{15}$ Y. Chenand C.-F. Wang, Characteristic modes: Theory and applications in antenna engineering (John Wiley \& Sons, 2015).

${ }^{16}$ Y. Chenand C.-F. Wang, "Hf band shipboard antenna design using characteristic modes," IEEE Transactions on Antennas and Propagation 63, 1004-1013 (2015).

${ }^{17}$ S. Genovesi, F. A. Dicandia, and A. Monorchio, "Excitation of multiple characteristic modes on a three dimensional platform," in 2017 11th European Conference on Antennas and Propagation (EUCAP) (IEEE, 2017) pp. 1769-1771.

${ }^{18}$ D.-W. Kimand S. Nam, "Systematic design of a multiport mimo antenna with bilateral symmetry based on characteristic mode analysis," IEEE Transactions on Antennas and Propagation 66, 1076-1085 (2017).

${ }^{19}$ M. H. Rabah, D. Seetharamdoo, M. Berbineau, and A. De Lustrac, "New metrics for artificial magnetism from metal-dielectric metamaterial based on the theory of characteristic modes," IEEE Antennas and Wireless Propagation Letters 15, 460-463 (2016).

${ }^{20}$ M. Capek, P. Hazdra, and J. Eichler, "A method for the evaluation of radiation q based on modal approach," IEEE Transactions on Antennas and Propagation 60, 4556-4567 (2012).

${ }^{21}$ M. H. Rabah, D. Seetharamdoo, and M. Berbineau, "Analysis of miniature metamaterial and magnetodielectric arbitrary-shaped patch antennas using characteristic modes: Evaluation of the $q$ factor," IEEE Transactions on Antennas and Propagation 64, 2719-2731 (2016).

${ }^{22}$ J. Eichler, P. Hazdra, M. Capek, and M. Mazanek, "Modal resonant frequencies and radiation quality factors of microstrip antennas," International Journal of Antennas and Propagation 2012 (2012).

${ }^{23}$ R. Martens, E. Safin, and D. Manteuffel, "Inductive and capacitive excitation of the characteristic modes of small terminals," in 2011 Loughborough Antennas 83 Propagation Conference (IEEE, 2011) pp. 1-4.

${ }^{24}$ D. Seetharamdoo, M.-h. Rabah, H. Srour, and M. Berbineau, "Method for improving the efficiency of an electrically small antenna," (2019), uS Patent App. 16/311,474.

${ }^{25} \mathrm{M}$. Cabedo Fabres, Systematic design of antennas using the theory of characteristic modes, Ph.D. thesis (2008).

${ }^{26} \mathrm{M}$. H. Rabah, Design methodology of antennas based on metamaterials and the theory of characteristic modes: application to cognitive radio, $\mathrm{Ph} . \mathrm{D}$. thesis, Lille 1 (2015).

${ }^{27} \mathrm{M}$. H. Rabahand D. Seetharamdoo, "Analysis and design of metamaterial structures using the theory of characteristic modes," in 2017 11th European Conference on Antennas and Propagation (EUCAP) (IEEE, 2017) pp. 2676-2680.

${ }^{28}$ R. Garbaczand D. Pozar, "Antenna shape synthesis using characteristic modes," IEEE Transactions on Antennas and Propagation 30, 340-350 (1982)

${ }^{29}$ M. M. Elseweand D. Chatterjee, "Characteristic mode analysis of microstrip patch shapes," in 2017 IEEE International Symposium on Antennas and Propagation 85 USNC/URSI National Radio Science Meeting (IEEE, 2017) pp. 2107-2108.

${ }^{30} \mathrm{~B}$. Yangand J. J. Adams, "Systematic shape optimization of symmetric mimo antennas using characteristic modes," IEEE Transactions on Antennas and Propagation 64, 2668-2678 (2015).

${ }^{31}$ E. FEKO, "Simulation software," (2014).

${ }^{32} \mathrm{R}$. Harringtonand J. Mautz, "Theory of characteristic modes for conducting bodies," IEEE Transactions on Antennas and Propagation 19, 622-628 (1971).

${ }^{33}$ M. C. Fabres, "Systematic design of antennas using the theory of characteristic modes," Universidad Politecnica Valencia , 118120 (2007).

${ }^{34}$ R. Harringtonand T.-H. E. Fields, "Hoboken," (2001).

${ }^{35}$ M. Gustafsson, J. Friden, and D. Colombi, "Antenna current optimization for lossy media with near-field constraints," IEEE Antennas and Wireless Propagation Letters 14, 1538-1541 (2014).

${ }^{36}$ M. Cismasuand M. Gustafsson, "Antenna bandwidth optimization with single frequency simulation," IEEE Transactions on Antennas and Propagation 62, 1304-1311 (2013).

${ }^{37} \mathrm{~W}$. Geyi, "A method for the evaluation of small antenna q," IEEE Transactions on Antennas and Propagation 51, 2124-2129 (2003).

${ }^{38} \mathrm{H}$. Benosmanand N. B. Hacene, "Design and simulation of double" s" shaped metamaterial," International Journal of Computer Science Issues (IJCSI) 9, 534 (2012).

${ }^{39}$ X. Chen, T. M. Grzegorczyk, B.-I. Wu, J. Pacheco Jr, and J. A. Kong, "Robust method to retrieve the constitutive effective parameters of metamaterials," Physical Review E 70, 016608 (2004). 
${ }^{40}$ D. Smith, D. Vier, T. Koschny, and C. Soukoulis, "Electromagnetic parameter retrieval from inhomogeneous metamaterials," Physical review E 71, 036617 (2005).

560

${ }^{41}$ A. Sihvola, "Metamaterials in electromagnetics," Metamaterialsso 1, 2-11 (2007).

${ }^{42}$ Y. Rahmat-Samii, "Metamaterials in antenna applications: Classifications, designs and applications," in Antenna Technology Small Antennas and Novel Metamaterials, 2006 IEEE International Workshop on (IEEE, 2006) pp. 1-4.

${ }^{43}$ H. Chen, J. Zhang, Y. Bai, Y. Luo, L. Ran, Q. Jiang, and J. A. Kong, "Experimental retrieval of the effective parameters of metamaterials based on a waveguide method," Optics Express 14, 12944-12949 (2006).

570

${ }^{44}$ X. Chen, B.-I. Wu, J. A. Kong, and T. M. Grzegorczyk, "Re-590 trieval of the effective constitutive parameters of bianisotropic metamaterials," Physical Review E 71, 046610 (2005).

${ }^{45}$ D. Seetharamdoo, R. Sauleau, K. Mahdjoubi, and A.-C. Tarot, "Effective parameters of resonant negative refractive index metamaterials: Interpretation and validity," Journal of applied physic\$95 98, 063505 (2005).
${ }^{46}$ R. O. Ouedraogo, E. J. Rothwell, A. R. Diaz, K. Fuchi, and A. Temme, "Miniaturization of patch antennas using a metamaterial-inspired technique," IEEE Transactions on Antennas and Propagation 60, 2175-2182 (2012).

${ }^{47}$ A. Erentokand R. W. Ziolkowski, "Metamaterial-inspired efficient electrically small antennas," IEEE Transactions on Antennas and Propagation 56, 691-707 (2008).

${ }^{48}$ P. Jinand R. W. Ziolkowski, "Metamaterial-inspired, electrically small huygens sources," IEEE Antennas and Wireless Propagation Letters 9, 501-505 (2010).

${ }^{49}$ J. L. Ethier, Antenna shape synthesis using characteristic mode concepts, Ph.D. thesis, Université d'Ottawa/University of Ottawa (2012).

${ }^{50}$ S. Kasapand P. Capper, Springer handbook of electronic and photonic materials (Springer, 2017).

${ }^{51}$ K. Schab, L. Jelinek, M. Capek, C. Ehrenborg, D. Tayli, G. A. Vandenbosch, and M. Gustafsson, "Energy stored by radiating systems," IEEE Access 6, 10553-10568 (2018).

${ }^{52}$ D. Nyberg, P.-S. Kildal, and J. Carlsson, "Radiation q and radiation efficiency of wideband small antennas and their relation to bandwidth and cut-off of spherical modes," (2007). 